\title{
El método etnográfico y su relación con el análisis de dominio
}

\author{
Manuel Alejandro Romero Quesada, Ania Rosa Hernández Quintana \\ Universidad de la Habana - UH, Cuba.
}

\section{REVIEW}

\begin{abstract}
Resumen
Analiza la relación teórico-conceptual del método etnográfico con el análisis de dominio. Se realizó un análisis documental explorando las categorías Análisis de Dominio y Método Etnográfico. Se obtuvo como resultado el análisis de los puntos de contacto entre el Análisis de Dominio y el Método Etnográfico desde un punto de vista epistemológico, metodológico y procedimental. Se concluye que el método etnográfico constituye una herramienta de investigación importante para escudriñar los convulsos escenarios socio-culturales que suceden a lo interior de las comunidades discursivas que componen a los dominios de conocimientos.
\end{abstract}

Palabras clave

Método etnográfico ; Análisis de dominio ; Cultura ; Sociología ; Antropología ; Ciencia de la Información

\section{The ethnographic method and its relationship with the domain analysis}

\begin{abstract}
This paper analyzes the theoretical and conceptual relationship of the ethnographic method with domain analysis. A documentary analysis was performed, exploring the categories of domain analysis and ethnographic method.It was obtained as a result: the analysis of the points of contact between domain analysis and the ethnographic method from an epistemological, methodological and procedural terms. It is concluded that the ethnographic method is an important research tool to scan the turbulent socio-cultural scenarios that occur within discursive communities that constitute the domains of knowledge.
\end{abstract}

Keywords

Ethnography method; Domain analysis ; Culture ; Sociology ; Anthropology ; Information Science

\section{Introducción}

Las características interdisciplinares de la Ciencia de la Información $(\mathrm{Cl})$ junto a la existencia de los enfoques paradigmáticos que permean las disciplinas informativas, posibilitan cada vez más la aplicación de métodos nacidos en otras áreas de las Ciencias Sociales.

A partir de los continuos cuestionamientos al paradigma cognitivo de la $\mathrm{Cl}$, se erigen las perspectivas paradigmáticas vinculadas a lo contextual, cultural y social. El análisis de dominio (AD), propuesto por Hjørland y Albrechtsen (1995) constituye una de esas principales propuestas teóricas dentro del enfoque socio-cognitivo en la Cl. Dicha propuesta devela escenarios que posibilitan la articulación con métodos sociológicos de investigación.

Es válido aclarar que la visión de paradigma de Hjørland sintoniza perfectamente con la de Tomas Kuhn, en donde los paradigmas son mutuamente excluyentes y el origen de un paradigma implica el deceso de otro. Por ende su propuesta parte de la ineficiencia del paradigma cognitivo imperante en la $\mathrm{Cl}$ hasta ese entonces. Si bien esta visión de paradigma encuentra sus cuestionamientos fundamentalmente en las Ciencias Sociales, algunos teóricos las continúan usando por ser esta un punto de vista bastante establecido. 
"Las tensiones entre los defensores de los diversos puntos de vista, los cuales incluyen los puntos de vista físico, cognitivo, social y lingüístico, han constituido puntos de discusión clave en la ciencia de la información. Los debates acerca de a cuál visión debería dársele primacía, han oscilado de acá para allá y todavía continúan. Los puntos de vista diferentes han sido los principales impulsores de la investigación en la ciencia de la información en diferentes momentos del siglo pasado. La visión física, la cual describe la información como una señal que puede transmitirse entre entidades, tales como los seres humanos y las máquinas, surgió de la teoría de la comunicación de Shannon (Shannon 1948). El punto de vista cognitivo, la moda dominante que comenzó a finales de la década de 1970 y se extendió hasta mediados de la década de 1990, se basa en la visión de que la percepción humana, los estados internos de conocimiento y las creencias, constituyen los medios para que se den las actividades informacionales individuales" (Mayernik, 2007)

Para Hjørland el cognitivismo no tiene en cuenta las estructuras del conocimiento que surgen como fenómenos sociales y específicos de un dominio pues este "concibe el conocimiento como estados mentales individuales en lugar de, o en oposición al conocimiento como proceso social o cultural o como producto social". Propone una visión alternativa basada en la teoría de la actividad que él llama "colectivismo metodológico". El colectivismo metodológico difiere de la visión cognitiva, por cuanto "considera los dominios del conocimiento, las disciplinas, u otras estructuras del conocimiento colectivo" (Hjorland 1997; Mayernik, 2007)

Estos evidentes puntos en contacto con enfoques sociológicos permiten imbricar teorías de la $\mathrm{Cl}$ con métodos de la Sociología o Antropología Social las cuales bien se adecuan al estudio de comunidades y culturas, y que además pueden proveer a las $\mathrm{Cl}$ de un "carácter innovador para tratar cuestiones y problemas que traspasan sus fronteras disciplinares." (Bicalho y Oliveira, 2011)

Uno de los métodos más utilizados en la actualidad dentro de las Ciencias Sociales es sin duda el etnográfico. La extrapolación de este método hacia áreas como la Educación, la Comunicación, los estudios sobre Internet y la Psicología, cada vez se hacen más frecuentes.

Por ello, atendiendo a la interdisciplinariedad mencionada y la existencia de enfoques paradigmáticos que influyen el quehacer científico dentro de la $\mathrm{Cl}$, se debe enfatizar la necesidad de desarrollar las investigaciones que apliquen el método etnográfico en el campo teórico vinculado a la información, para la construcción de un conocimiento sociológico necesario dentro de la Cl. La Sociología y la Antropología Social suponen derroteros por los cuales la $\mathrm{Cl}$ deberá transitar, acoplando y/o modificando sus discursos y prácticas tradicionales. (Bufrem y Santos, 2009)

Por todo ello, este artículo propone sugerir un modesto acercamiento, a lo que concierne al método etnográfico aplicado a uno de los supuestos teóricos más importantes del llamado paradigma social, el AD de Hjørland (1995), y de esta manera promover que los profesionales de la información apliquen métodos de investigación cualitativa, específicamente de corte etnográfico, los cuales requieren de mayor aplicación en la actualidad.

\section{Análisis de dominio y método etnográfico, algunos escenarios convergentes.}

"El paradigma analítico (1) del dominio es una aproximación teórica a la $\mathrm{Cl}$, el cual establece que la mejor manera para entender la información en la $\mathrm{Cl}$ es a través del estudio de los dominios de conocimiento como comunidades discursivas, las cuales son parte de la división social del trabajo." (Hjørland y Albrechtsen, 1995)

De esta manera se hace evidente que el AD sintoniza con el espíritu de las investigaciones sociales, como muestra de clara avenencia con el referido enfoque socio-cognitivo y busca demostrar que la esencia de la $\mathrm{Cl}$ está en “(...) los movimientos sociales en la búsqueda de un proyecto humanista alternativo, y particularmente en la esfera científica, en el reconocimiento del ser humano como sujeto histórico en mutua interrelación con la naturaleza y la sociedad." (Vega y cols., 2009)

Es por ello que la $\mathrm{Cl}$ deberá girar su colimador hacia el $\mathrm{AD}$, el cual es perfectamente aplicable en diferentes escenarios de la investigación en $\mathrm{Cl}$, pues son "La organización del conocimiento, la estructura, los patrones de co-operación, las formas del lenguaje y la comunicación, los sistemas de información y los criterios de relevancia, reflejos de los objetos del trabajo de esas comunidades y de su papel en la sociedad" (Hjorland y Albrechtsen 1995)

El necesario abordaje de lo socio-cultural dentro del AD y las necesidades de describir las particularidades acaecidas al interior de las comunidades abre las puertas a migraciones conceptuales y metodológicas de otras 
Ciencias Sociales, encargadas de compartir la responsabilidad de esclarecer las complejidades de los procesos de uso y acceso de la información y el conocimiento y las condicionantes socio-culturales que estas poseen.

La Sociología y la Antropología Social constituyen ciencias sociales que analizan las interacciones de los individuos en sus comunidades y ámbitos sociales a través fundamentalmente del método etnográfico. "Dicha metodología con sus referentes en la Antropología (2) Cultural o Social (...) [es utilizada] por los sociólogos como respuesta a la crítica planteada contra el uso de los métodos positivistas para los estudios sociales." (Mosquera, 2008)

El AD comprende a los dominios de conocimientos como sistemas que, en sus múltiples relaciones y articulaciones, conforman sus marcas estructurales. Bajo esta visión, el AD es también un aparato teóricometodológico que describe las bases socioculturales de las interacciones informacionales en cualquier estructura específica de los dominios de conocimiento.

Los presupuestos sociológicos del AD develan teorías que proporciona un fundamento epistemológico firme para la investigación en las Csl, para estudiar las diferentes prácticas humanas, sus interacciones sociales, estratificaciones, múltiples niveles y maneras de desarrollarse en el tiempo, tomando los sistemas de actividades colectivas como la unidad de análisis, en general, y el conocimiento creado mediante la acción humana, en particular. (Mayernik, 2007; Wilson, 2006; Hjørland y Nicolaisen, 2005)

El enfoque social del AD define al conjunto de las disciplinas informativas como una ciencia social y promueve sus perspectivas socio-psicológica, sociolingüística y socio-cognitiva, desde el entendimiento que son los actores sociales los que estudian los dominios de conocimiento y son las asunciones sociológicas las regentes en los estudios de interacciones sociales, construcciones sociales del conocimiento y develación de las bases socioculturales de las comunidades discursivas. (Hjørland y Albrechtsen, 1995)

Es por ello que el método etnográfico es perfectamente aplicable en las $\mathrm{Cl}$ y por consiguiente al AD. Este constituye una teoría que demanda aplicaciones, visiones y soluciones desde perspectivas sociológicas. Su inminente carácter sociocultural le permite coexistir precisamente en las fronteras teóricas entre la Sociología y las Cl. Por ello el método etnográfico, al devenir herramienta funcional para efectuar el proceso analítico de dominio, constituye un área en la cual los profesionales de la información deben explorar y conocer a profundidad.

Por ende la incorporación de teorías que profundicen en el complicado orden social se hace cada vez más importante para las disciplinas informativas, pues que mejor sustrato teórico que el devenido de la Sociología, ciencia que se pregunta el porqué de la existencia y el orden social, además de cuáles son los significantes y significados sociales de las comunidades. Obviamente la Sociología y su teoría poseen escenarios de investigación aproximados a la $\mathrm{Cl}$. Es la Sociología de la información ese punto convergente, la frontera existente entre la Sociología y las Cl. (Hjørland, 2005)

La $\mathrm{Cl}$ en su discurrir paradigmático se ha formado bajo fuertes bases interdisciplinares y transdisciplinares. La adquisición de conceptos de otras áreas del saber marca el cuerpo teórico de dicha Ciencia. Entonces existe un apoyo epistemológico y tradicional hacia la adopción de elementos de la teoría sociológica. La aproximación de estos dos campos posibilita -por el carácter multidisciplinar de la $\mathrm{Cl}$ - la materialización de esta relación mediante la Sociología de la información expone escenarios desde los cuales se hace posible imbricar el AD y el método etnográfico. O sea que si es posible la relación $\mathrm{Cl}$ - Sociología, también lo es la relación entre sus teorías y métodos. El etnográfico no queda exento de dicha relación.

\section{El método etnográfico y su escenario histórico-teórico}

La etnografía se encarga de develar los significados de las acciones emprendidas por las personas dentro de sus grupos sociales, aportando desde una visión holística la percepción más convincente para la indagación y la comprensión de las interacciones e interrelaciones sociales.

"Etimológicamente, el término etnografía significa la descripción (grafé) del estilo de vida de un grupo de personas habituadas a vivir juntas (ethnos). Por tanto, el ethnos, que sería la unidad de análisis para el investigador, no sólo podría ser una nación, un grupo lingüístico, una región o una comunidad, sino también cualquier grupo humano que constituya una entidad cuyas relaciones estén reguladas por la costumbre o por ciertos derechos y obligaciones recíprocos." (Martínez, 2005) 
Tanto la Escuela de Chicago como las investigaciones de Malinowski (3) sobre los nativos de la zona occidental de Nueva Guinea conforman los puntos de partida de este quehacer científico que utiliza el método etnográfico como herramienta metodológica por excelencia. (Murillo y Martínez, 2010; Mosquera, 2008)

Este método se aplica con el objetivo de describir la cultura, con una participación de la investigadora o investigador en la vida diaria de un grupo cultural definido sobre un período de tiempo prolongado; describir la cultura como conocimiento compartido y entendimiento de sentido común de los miembros de un grupo que determinan un comportamiento apropiado en tal escenario. (Domínguez, 2007; Mosquera, 2008; Muela 2004; Turpo, 2008)

Al aplicar el método etnográfico, el investigador deberá conocer y reconocer cuáles son las unidades de análisis que le ocupan, además de una previa delimitación de objetivos y preguntas que guiarán el discurrir investigativo y que "se considera significativo dirigir a un cierto fenómeno sociocultural, un conjunto de preguntas que delimita el ámbito de lo observable y de lo no observable, que siempre se hacen desde una perspectiva teórica concreta y que los antropólogos equiparan comúnmente a la cultura." (Jociles, 1999)

El concepto de cultura es una especie de rector teórico en cuanto a temas de desarrollos sociales. La noción de cultura ha pasado por distintos estadios conceptuales atendiendo a la evolución del pensamiento y las visiones científicas. "La cultura tiene un significado restringido y otro amplio; el restringido alude a la mente, gustos y modales refinados, esto es, a la condición de preparación e ilustración; el sentido amplio, sugiere que la cultura es el conjunto de recursos acumulados, materiales e inmateriales, que la sociedad hereda, usa, produce, aumenta y transmite."(Meneses, 2005)

Desde el punto de vista antropológico, y fundamentalmente en sus inicios, la cultura era entendida como un "todo complejo que incluye el conocimiento, las creencias, el arte, la moral, el derecho, las costumbres y cualesquiera otros hábitos y capacidades adquiridos por el hombre en cuanto miembro de la sociedad." Tylor (1871 cp. Basail y C.Álvarez, 2004).

Posteriormente y producto de la necesidad de estudiar e interpretar a fondo la cultura, esta noción adquiere dentro de la Antropología otras connotaciones, convirtiéndose en "esquemas históricamente transmitidos de significaciones representadas en símbolos, un sistema de concepciones heredadas y expresadas en formas simbólicas con las cuales los hombres comunican, perpetúan y desarrollan su conocimiento y sus actitudes frente a la vida." (Geertz, 1986)

Por ende la cultura, como escenario semiótico, permite que los antropólogos funjan como lectores culturales, decodificadores e interpretadores de las significaciones en las que se inserta el ser humano en su interactuar social. De esta manera, la cultura adquiriría características textuales, conformado por sistemas de símbolos con significados particulares a cada comunidad. (Geertz, 1986; Nivón y Rosas, 1991)

Otro de los conceptos imprescindibles para abordar el método etnográfico es sin dudas el concepto de campo. El término "campo" se refiere al lugar en el que se observa la cotidianidad de una comunidad para recoger sobre el terreno las informaciones que, directamente, les proveen los propios individuos. El trabajo etnográfico es, por excelencia, el trabajo de campo; el método etnográfico es el que guía las acciones en torno al campo cultural que constituyen a las distintas comunidades. (Moreno, 2007)

\section{El método etnográfico y su escenario procedimental}

El proceso etnográfico no responde a directrices estrictas pues se afectaría la apropiación de información de un medio convulso, dinámico y multi-causal, por lo que más bien depende de las situaciones que se van generando en el escenario natural de las comunidades.

"Uno de los rasgos predominantes del proceso etnográfico y que define las fases de indagación en esta metodología, es el carácter emergente de sus etapas, las cuales no están claramente definidas como en otros tipos de investigación. Sin embargo, es necesario que el investigador tenga un marco inicial sobre el qué, quién, dónde, cuándo, por qué y cómo acerca de la realidad a estudiar." (Mosquera, 2008)

A continuación se expondrán los rasgos de las principales fases de aplicación del método etnográfico:

- La primera de las fases de este proceso responde a la delimitación y selección del objeto de estudio, así como el trazado de los objetivos y preguntas de investigación. 
Además se establecerán los tiempos y lugares necesarios para la pesquisa. (Murillo y Martínez, 2010; Calventus, 1996; Covarrubias, 1998)

Para al AD esta fase sería primordial, pues permitirá delimitar la comunidad desde elementos como la interacción con la información o patrones culturales en la adquisición y generación de conocimientos, por ejemplo. Cuando se habla de dominios de conocimiento es lógico pensar en comunidades extensas, pero la aplicación del método etnográfico deberá circunscribirse a determinados contextos que tengan la posibilidad de ser observados y descritos.

- La segunda fase de este proceso responde a un momento empírico-analítico. Constituye el punto central de la investigación, pues es el proceso de recolección de datos en el campo. Se realiza la llegada y entrada al contexto de ocurrencia así como selección de informantes claves, etc. Además se lleva a cabo un registro descriptivo de lo que sucede en el contexto de ocurrencia. La información que se busca es aquella que más relación tenga y ayude a descubrir mejor las estructuras significativas que dan razón de la conducta de los sujetos en estudio. (Martínez, 2005; Calventus, 1996)

Luego de haber delimitado y contextualizado la comunidad hay que insertarse en ella para realizar los escrutinios pertinentes a los objetivos y preguntas de investigación. Este constituye un momento que requiere de una preparación previa pues es necesario interactuar con un particular discurso y poseer referentes culturales y cognoscitivos que sintonicen con esa comunidad.

Esto permitirá que el investigador no tenga lagunas conceptuales que afecten el proceso de observación y descripción por lo que debe realizar un proceso de análisis documental y discursivo en busca de referentes conceptuales, visiones paradigmáticas, terminológicas y semióticas. Este proceso es conocido entre los investigadores que aplican el método etnográfico como análisis de datos secundarios, los cuales constituyen todo aquellos datos que no se obtienen directamente del campo de investigación.

Aunque la obtención de datos secundarios resulta importante, esta etapa del proceso etnográfico está marcada por "el trabajo de campo", principal atributo y esencia de toda investigación que utilice el método etnográfico y mediante el cual se obtienen los datos primarios vinculados a las comunidades a estudiar.

"El trabajo de campo se caracterizaba, tradicionalmente, por la estancia prolongada de un investigador en una comunidad pequeña con la que no compartía la misma cultura, interfiriendo de forma mínima en la recopilación de datos." (Sánchez, 2003)

El acercamiento a esos campos deviene en momento decisivo y complejo para el investigador etnográfico. Tanto las dispersiones de lo observable como la ambigüedad de lo observado pudieran desconcertar un tanto al investigador en su primera visita al campo.

"En los primeros momentos de la investigación, las observaciones pueden no estar del todo centradas, y es preciso que el investigador vaya aprendiendo en los primeros días quién es quién y construir un esquema o mapa de los participantes en el lugar y un mapa en la distribución física del escenario." (Rodríguez, Gil y García, 1996)

En ocasiones, aunque el investigador se encuentre en un determinado campo, este se aísla del mismo al ubicarse en sus propios paradigmas, conceptos, metodologías e instrumentos y pretendiendo entender y juzgar desde su posición etnocentrista. El entendimiento del otro deberá llevarse a cabo desde el entendimiento de las categorías, leyes, representaciones y en general la cultura en la que ese otro está inmerso. (Campo, 2001)

Por ende, la categoría conceptual "otredad" constituye una de las nociones más importantes a tener en cuenta por el investigador en el trabajo de campo. De hecho, entender la otredad es la finalidad de dicho trabajo. La comprensión del otro, sin posiciones etnocentristas, posibilita estudios etnográficos que reflejan las culturas de las comunidades de manera más diáfana y apegada a la realidad.

Para el $A D$, el respeto a la otredad es fundamental pues cada comunidad tiene sus propios métodos de acercarse a los fenómenos objetos de estudios y de interactuar con la información. El esfuerzo por comprender al otro, no cuestionarlo ni juzgarlo desde leyes que no les pertenezcan y utilizar un conjunto de técnicas que le permitan adentrarse en el campo y recuperar la mayor y mejor cantidad de información es sumamente importante. 


\section{El método etnográfico y sus técnicas}

La extracción de información a través del trabajo de campo requiere de disímiles técnicas que canalicen los datos y se usan indistintamente dentro del proceso etnográfico para ir descifrando la composición de lo estudiado.

A continuación se expondrán las técnicas comúnmente utilizadas:

\subsection{Observación participante}

Es la técnica primordial del método etnográfico, una especie de plataforma que ayuda a entretejer a la reflexividad del investigador con el objeto a estudiar. Su principal objetivo radica en el registro y la descripción detallada de lo observado. Es importante al observar tener en cuenta cómo se observa, pues lo ideal es modificar lo menos posible la situación objeto de estudio, adoptando un modo de actuar si bien participativo, pero no injerencista. La observación deberá estar no solo asociada al sentido de la vista, pues el investigador deberá verter en esta observación participante todos los sentidos posibles en busca de percibir del campo la mayor cantidad de sensaciones. (Covarrubias, 1998; C. Álvarez, 2008; Urraco, 2007; Whitehead, 2005)

Spradley (1980 c.p. Whitehead, 2005) determina cuatro niveles de participación para la observación:

- Participación pasiva: es cuando el investigador está presente en el escenario pero su participación es muy limitada.

- Participación moderada: existe mayor participación que en la pasiva, pero aún es limitada la participación con el objetivo de mantener un balance entre las visiones del investigador y las del investigado.

- Participación activa: el observador no solo busca qué están haciendo los otros sino más bien realiza un aprendizaje completo del escenario y su cultura, reglas y comportamientos.

- Participación completa: es cuando el observador está completamente envuelto en todos los aspectos del escenario adquiriendo un rol dentro de este.

Para el $A D$ la participación activa resultará un tanto compleja, por lo que se deberá desarrollar una fuerte estrategia de observación que le permitan al investigador irse apropiando paulatinamente de los códigos discursivos y otros elementos que lo vayan guiando en el campo.

\subsection{Las entrevistas cualitativas o en profundidad}

Es la técnica a través de la cual el investigador se acerca al investigado. Su ventaja es que estimula el flujo de los datos y que ofrece una información personal que de otro modo sería imposible conocer. Permite establecer relaciones de empatía entre el investigador y el informante. Aunque en su aplicación las intenciones están orientadas hacia lo individual es precisamente desde esa posición intimista que se intenta entender cómo entienden otros las situaciones sociales y comunes, posibilitando triangular las visiones de varios informantes llegando a consensos de los puntos de vistas. (Covarrubias, 1998; C. Álvarez, 2008; Jociles, 1999)

Las entrevistas permitirán el análisis discursivo de los integrantes de las comunidades. Captar códigos discursivos particulares de la comunidad en cuestión y sus múltiples relaciones con las estructuras de conocimiento perteneciente a sus dominios, dotan a esta técnica de un valor incalculable. Las entrevistas arroparán al investigador de aquellas representaciones discursivas presentes en la cultura de dichas comunidades. (Whitehead, 2005) 


\subsection{Discusión de grupos}

Es fundamental para entender las representaciones sociales de las comunidades y se utiliza en busca del consenso. La discusión de grupo constituye una toma de contacto con la realidad en las que los miembros del grupo colaboran en la definición y auto-comprensión de sus propias realidades sociales. La voz conjunta que de esta discusión emana puede ser la validación grupal de la interpretación de investigador. (Jociles, 1999; García, Ibáñez, Alvira, 1994)

Matus y Molina (2006) advierten las siguientes instancias para la correcta implementación de esta técnica:

- Diseño, estructura y formación del grupo: la cantidad de personas que integran el grupo deberá oscilar entre 5-10, menor a esta cantidad sesgaría la riqueza de las interacciones buscada en investigaciones de esta índole. La selección de los integrantes responderá a los objetivos de la investigación y a la articulación que entre estos pueda existir en función de las necesidades informativas del investigador.

- Proceso y desarrollo del grupo de discusión: El encuentro se pactará en un lugar que represente un ambiente tranquilo y distendido. Se sugiere que la disposición grupal sea de forma circular con marcada posición del conductor del grupo .El investigador y/o conductor del grupo deberá presentar los objetivos del debate y de la investigación pero sin emitir juicios que coaccionen al grupo para que así se expresen libremente.

\section{Análisis de los datos y escritura etnográfica}

Una vez aplicadas las técnicas de recolección de datos a un determinado campo de acción se hace indispensable materializar el análisis e interpretación de dichos datos para posteriormente llegar a la escritura etnográfica, la actividad colofón del proceso etnográfico. Este momento analítico-escritural constituye una fase mutuamente relacionada e inseparable.

"Es mentira que el análisis y la redacción sean etapas separadas de una investigación. Al escribir se siguen atando cabos que han quedado sueltos; y aun cuando se hagan por anticipado elaborado análisis de los datos, se lleva a cabo uno adicional, pues al redactar se reflexiona sobre lo que se está escribiendo. Todavía al corregir el borrador se afinan ideas, se desechan hipótesis y se piensan otras."(García, 2011)

El análisis de los datos constituye la materia prima de lo que posteriormente será el texto etnográfico (4). Ese análisis está presente en cada aplicación de las anteriores técnicas en el campo de estudio. Su objetivo es reconocer, elaborar e identificar las categorías de significación que emanan del grupo estudiado. Es un proceso complejo, dinámico y recursivo que sigue un principio de acción-reflexión-acción y genera elementos analíticos los cuales pueden ser sometidos a nuevos análisis a partir de nuevas observaciones. (Murillo y Martínez, 2010; Gil, Henao, y Peñuela, 2004)

E. Álvarez (2010) propone tres momentos en los cuales se desarrolla el análisis de los datos obtenidos en el campo:

\section{a) Reflexión analítica sobre los datos}

A la par que el etnógrafo recoge los datos, realiza una tarea de reflexión que es fundamental para la organización y selección de los mismos. El etnógrafo juega un papel "centralizador" en todo el estudio. Su mente archiva y desecha, recoge y analiza, reflexiona sobre lo vivido, lo sentido, lo pensado, los datos recogidos.

\section{b) Selección y reducción de datos}

Reducir los datos con los que se va a trabajar finalmente no es una tarea sencilla, pues implica dejar a un lado gran cantidad de material ¿Cómo realizar esa selección? Los intereses del estudio tienen que guiar ese proceso.

\section{c) Organización y categorización de los datos}

Permanentemente el etnógrafo, en su esfuerzo por dar sentido a los datos recogidos, genera y regenera categorías explicativas en las que agrupa los significados más relevantes recogidos. Esta 
categorización no es una tarea sencilla ni definitiva, pues se encuentra sometida a permanente revisión y transformación, en cuanto aparece un nuevo dato que nos hace repensarla. Además, con el objeto de llegar a formular las conclusiones, las categorías deben reagruparse formando redes que proporcionen información sobre las relaciones existentes entre las diferentes unidades de significado.

Luego de materializar los análisis a los conjuntos de datos obtenidos en el campo, se comienza a conformar el texto, producto final del proceso etnográfico. Elaborar ese texto suele ser una tarea ardua, compleja y de mucha responsabilidad pues requiere tiempo de lecturas y relecturas de las notas de campo, el re-análisis de los datos, las observaciones y las entrevistas. El propio proceso de redacción es una actividad dura, rigurosamente disciplinada, que exige dedicación, tranquilidad, optimismo, reflexión permanente y sobre todo tiempo. (C. Álvarez, 2008)

El texto etnográfico constituye la plasmación escrita del resultado de las aplicaciones de disimiles técnicas en el trabajo de campo, junto con la posición reflexiva e interpretativa del investigador en los procesos de descripción de símbolos y representaciones culturales de las comunidades. Es un proceso de confluencia entre las anotaciones sobre lo observado y las ideas del observador. La representación escrita de los conocimientos obtenidos a través de la creación de dicho texto constituye la finalidad del proceso etnográfico. (Ardèvol, Adolfo, y Domínguez, 2008; Sánchez, 2003)

Las principales fuentes para la conformación del texto etnográfico son según Sánchez (2003):

- Textos que produce el etnógrafo (cuadernos de notas, diario, cuaderno de campo y todo tipo de notas y observaciones)

- Textos escritos por "los otros" (cartas, diarios, todo tipo de materiales de archivos, periódicos, documentos de instituciones, etc.)

- Objetos de la cultura material del lugar

- Grabaciones de audio/vídeo

- Fotografías

- Nosotros mismos, es decir, nuestras notas mentales

El contenido del texto etnográfico será consecuente con las respuestas a las preguntas de la investigación y se adecuara con los objetivos planteados al inicio. El estilo de escritura dependerá en gran medida de las particularidades, tanto del escritor como del público al que va dirigido. Fundamentalmente suelen existir dos destinatarios de dichos textos, la comunidad que estudia o la comunidad estudiada. (García, 2011)

Existe un problema discursivo importante a tener en cuenta a la hora de la redacción del texto etnográfico y se refiere a lo que muchos antropólogos han denominado como la relación Emic-Etic. Dicha relación responde -en primera instancia- al discurso de los miembros de la comunidad estudiada y,por otra parte, al discurso propio del investigador acerca de la realidad cultural estudiada. El relato Emic es el que se genera a partir de las opiniones y vivencias del observador cuando se convierte en un nativo más. El relato Etices el que permite ver la cultura con la mirada que realiza un observador externo a ella. Esta relación condicionará sin duda las posturas narrativas del texto etnográfico. Las intenciones y finalidad del texto deberán determinar la yuxtaposición o no de los relatos Emic o Etic. (C. Álvarez, 2008)

La aplicación del método etnográfico al AD deberá generar textos etnográficos que logren vincular las posiciones de los destinatarios y de los investigadores. Es necesario escribir un texto que cuente con la validación de los contenidos por parte de la misma comunidad estudiada.

\section{El investigador etnográfico y sus características}

El investigador que aplique el método etnográfico deberá poseer un grupo de características especiales y un conjunto de conocimientos y habilidades específicas que le permitan encarar las realidades acaecidas en una determinada comunidad en su "multiplicidad de estructuras conceptuales complejas, muchas de las cuales están 
superpuestas o en-lazadas entre sí, estructuras que son al mismo tiempo extrañas, irregulares, no explícitas, y a las cuales el etnógrafo debe ingeniarse de alguna manera, para captarlas primero y para explicarlas después." (Geertz, 1986)

Por ende los aspectos éticos del investigador resultan un peldaño el cual se debe vencer antes de someterse a la investigación cualitativa en general y la aplicación del método etnográfico en particular. La ética se vislumbra en disímiles direcciones dentro de la investigación, creando relaciones éticas con el sujeto a investigar, la propia investigación y el destinatario de la investigación.

"Las propias actividades del etnógrafo, en tanto investigador, están abiertas a lo desconocido por tratarse de las prácticas de un saber en gestación, y por tanto, poseen un fin incierto". (E. Álvarez, 2010) Aun así debe preestablecer ciertas estrategias que no deben ser obviadas, por ejemplo:

- El extrañamiento

"El extrañamiento guarda una estrecha relación con dos aspectos básicos: (1) el desarraigo de abandonar los espacios que habitualmente uno frecuenta, y (2) el afrontamiento de una situación desconocida, que además ha de ser examinada para ser comprendida. Es una actitud vital a lo largo de todo el proceso de investigación, no únicamente en los primeros momentos y guarda una estrecha relación con la capacidad del investigador para afrontar con sensación de asombro cada observación realizada, por vulgar, familiar o rutinaria que parezca a simple vista, pues profundizando en su significado puede resultar muy reveladora para la investigación." (C. Álvarez, 2008).

"Esa actitud conduce a entender cualquier forma de institución humana como el resultado de prácticas de convencionalización. El sustrato conceptual de esta noción se encuentra en el concepto de cultura como conjunto más o menos sistemático de convenciones" (Díaz, 2010).

Constituye un sustrato importante en la investigación no solo etnográfica, sino también en las Ciencias Sociales en general. Su propósito es la búsqueda de los planos más objetivamente cualitativos a partir de los fenómenos estudiados. El extrañamiento sistematizado es la experiencia específica que permite la generación de un tipo de conocimiento sobre el ser humano y cualquiera de sus producciones, siempre y cuando este conocimiento sea obtenido una vez despojado el etnocentrismo del investigador y adoptado los juicios valores y creencias de la comunidad estudiada. Es entonces que dichos productos y conocimientos pueden ser vistos como componentes que a su vez constituyen al productor. (E. Álvarez, 2010)

- La ignorancia docta

"Esta ignorancia es docta en tanto reconoce límites y actúa como un motor para el aprendizaje, la investigación y el deseo de saber. Se diferencia entonces de lo que podríamos llamar una "ignorancia necia" donde no opera la curiosidad y hay, en cambio, un conformismo con lo que ya se sabe, sin someterlo a discusión ni contrastarlo con la práctica. La ignorancia docta puede ser definida como una disposición para interrogar, para reconocer la dificultad en acceder a la verdad y aun así insistir en su búsqueda. El principal propósito y lo que determina el trabajo del indagador desde una perspectiva de la docta ignorancia, no es devolverle al otro una descripción que le es ajena, ni trasmitirle el conocimiento teórico de su situación, es promover que el sujeto investigado llegue a su propio saber" (Gil, y cols., 2004).

Este requerimiento es importantísimo pues permite al investigador no contaminar - al menos no en un alto por ciento- la información y el conocimiento obtenido en el campo.

- Ser uno más

Conceptos que aluden a conductas específicas, tales como approche y rapport tienen carta de ciudadanía como legítimas estrategias de seducción social, destinadas a que el investigador pueda ser aceptado venciendo la potencial resistencia de las comunidades. 
El etnógrafo no sólo tiene que extrañarse con lo ajeno, además tiene que tratar de integrarse parcialmente en el campo que estudia. Los procesos de acercamiento e integración constituyen un arma de vital importancia para el investigador. Lo que el investigador quiere deberá paulatinamente lograrse estableciendo relaciones abiertas con los miembros del grupo que estudia.

La selección de los informantes es uno de los puntos delicados en el proceso investigativo. En el momento en el que el investigador está intentando ser uno más simultáneamente deberá ir chequeando las posibilidades de quienes pueden ser potenciales informantes. En las aplicaciones del método etnográfico, se hace necesario utilizar distintos tipos de informantes para aproximarse a una realidad determinada y desde la pluralidad de variados puntos de vistas. Las propias estrategias para establecer el rapport ayudarán a la selección de los informantes. Como esta se adecúa al momento de acercamiento y propósito de ser uno más de la comunidad, entonces la selección de los sujetos informantes deberá llevarse a cabo con empatía, siguiendo los códigos de conducta establecidos, dominando el mundo simbólico y su lenguaje particular de la comunidad en estudio, estableciendo el compromiso y negociación. (Bartolomé, 2003; Mosquera, 2008).

- Reflexividad

"En la vigilancia constante de la reflexividad, el etnógrafo intenta abrirse lo más posible a la novedad de las otredades, a la captación de la expresión de lo diverso y heterogéneo en su multiplicidad, gracias a no dejar en suspenso y no tematizadas las bases epistemológicas y ontológicas que constituyen los cimientos del análisis científico. Pertrechado de herramientas como todo investigador, intenta no condicionar lo más posible o al menos, llevar registro e integrar las modificaciones que inevitablemente su participación genera." (E. Álvarez, 2010).

Estar siempre dispuesto a cuestionarse y reflexionar sobre lo que se va obteniendo e interpretando en el campo es una cualidad del investigador. Su teoría y conjeturas acerca de la investigación deben estar abiertas al cambio constante y la revisión permanente, es decir debe de estar dispuesto y atento a esperar lo que proviene del otro y su contexto.

El proceso de formalización que realiza el indagador tiene un carácter cíclico, evolutivo, es un punto de partida y de llegada que abre nuevas posibilidades para comenzar de nuevo. Es un intento de aprehender la experiencia de su indagación frente al objeto que observa (...) No existen conocimientos absolutos que le guíen, es su formación y su intuición la que le permite establecer su hacer." (Gil, cols., 2004).

Además de las referidas estrategias el investigador se auxilia de un conjunto de herramientas que le sirven de instrumentos para el registro de la información obtenida en el trabajo de campo y además facilitan los procesos analíticos y de conformación del texto etnográfico. Entre las herramientas más destacadas se encuentran:

\section{- Diario de campo}

Permite al etnógrafo registrar lo observado, sus formas de observación y el proceso mismo de su reflexividad sobre los acontecimientos de los que están participando cotidianamente. (Covarrubias, 1998).

- Libreta de campo

Está dirigida a poner en claro la relación que el etnógrafo tiene en determinado momento con el objeto (se refiere a como se construye al objeto, como se determinó, como se va a trabajar). (Covarrubias, 1998, Sajek, 1990) Fichas de registro: De carácter individual, donde se anotan datos de cada informante como nombre, lugar y fecha de nacimiento, región, nivel escolar. (Covarrubias, 1998) Grabadora de audio y/o vídeo: La mediación de las tecnologías en la interacción con el campo es común en las prácticas etnográficas actuales. (Ardèvol y cols., 2008; Covarrubias, 1998).

\section{- Fichas de registro}

De carácter individual, donde se anotan datos de cada informante como nombre, lugar y fecha de nacimiento, región, nivel escolar. (Covarrubias, 1998). 
- Grabadora de audio y/o video

La mediación de las tecnologías en la interacción con el campo es común en las prácticas etnográficas actuales. (Ardèvol y cols., 2008; Covarrubias, 1998).

La imbricación de estas herramientas con las categorías antes expuestas conforma el conjunto de conocimientos que debe poseer un investigador que aplica el método etnográfico. Todo esto, unido a las comprensiones de las significaciones de lo que es etnografía y las definiciones de su proceso y técnicas a emplear, conformarán el más elemental derrotero de la aplicación del método etnográfico al $A D$.

\section{Algunas razones sobre la importancia en la relación método etnográfico-análisis de dominio}

La información constituye una dimensión o artefacto cultural presente en la historicidad de los sujetos, en el funcionamiento de las estructuras y relaciones sociales de los mismos. La información es un fenómeno social y por ende de carácter y articulación cultural. (Nascimento y Marteleto, 2005, Gómez, 2010)

"En la $\mathrm{Cl}$ se acredita que la información es un elemento de la cultura, pues (...) adquiere sentido al ser comunicada para producir conocimiento (...) Los estudiosos y profesionales del campo de la información (...) asocian la información al conocimiento y a la comunicación y, por tanto, a la cultura". (Bufrem y Santos, 2009)

Aunque el término cultura no sea explícito en el aparato teórico-conceptual primigenio de Hjorland, las estructuras de conocimientos, teorías, disciplinas, discursos privativos, prácticas, objetos de estudios e información, constituyen elementos y símbolos culturales que están en la comprensión del AD. Al aplicar el método etnográfico al $A D$, la unidad de análisis también será la cultura, en este caso la de las comunidades discursivas o los dominios de conocimiento, aunque los objetivos de la investigación estarían orientados aquellos significantes culturales vinculados a la información y el conocimiento.

EI AD que utilice el método etnográfico encontrará en las culturas informacionales, académicas e incluso organizacionales sus unidades de análisis, y se podrán develar aspectos importantes sobre los procesos de acceso, generación, consumo de información, así como las relaciones entre estos y los discursos y conocimientos de determinadas comunidades.

La deconstrucción de dominios utilizando el método etnográfico permitirá la elaboración de inferencias sobre esquemas de organización del conocimiento en diferentes escalas sociales, así como el comportamiento humano en entornos informacionales. Además, abrirá oportunidades en áreas como los estudios de usuarios, la gestión de servicios y productos de información, las consultorías y la mercadotecnia, pues todas requieren estudiar las comunidades pertinentes.

\section{Conclusiones}

La Cl está permeada por paradigmas y enfoques que potencia las visiones sociales y contextuales para estudiar y entender todo proceso informativo. La necesidad de migraciones de aparatos teóricos de otras ciencias sociales a la $\mathrm{Cl}$ encuentra, fundamentalmente en lo que respecta a la sociología y antropología social, escenarios compartidos. La Sociología y Antropología abren un espectro teóricos-procedimental que la $\mathrm{Cl}$ deberían conocer y aplicar.

El método etnográfico constituye herramienta de investigación importante para escudriñar los convulsos escenarios socio-culturales que suceden a lo interior de las comunidades. El reconocimiento por parte del AD de la categoría "cultura" aportará a este un importante referente teórico y elemento definitivo e intrínseco de las comunidades, sus acciones y representaciones sociales.

El investigador etnográfico deberá potenciar sus referentes teóricos, técnicas, responsabilidades éticas y cualidades, sin las cuales el proceso etnográfico sería ineficiente desde el punto de vista informacional. La utilización del método etnográfico aportará indiscutiblemente descripciones culturales de las comunidades discursivas. 


\section{Notas}

1. Aunque Hjørland y Albrechtsen (1995) se refieran al análisis de dominio como paradigma, esta investigación lo asimila como una propuesta teórica, pues este no constituye ese paraguas epistemológico que influye el desarrollo teórico-práctico dentro de la $\mathrm{Cl}$.

2. Gran parte de la investigación antropológica se basa en trabajos de campo llevados a cabo con diferentes culturas. Entre 1900 y 1950, aproximadamente, estos estudios estaban orientados a registrar cada uno de los diferentes estilos de vida antes de que determinadas culturas no occidentales experimentaran la influencia de los procesos de modernización y occidentalización.

3. Antropólogo británico de origen polaco, considerado el fundador de la escuela funcional de antropología, defendió que las instituciones humanas deben analizarse en el contexto general de su cultura, a través de lo que él denominó como trabajo etnográfico.

4. Texto etnográfico, es la definición general del producto escriturado final de la aplicación del método etnográfico. Suelen llamarse monografías, ensayos, informes, etc.

\section{Bibliografía}

Aguirre J, J.C. (2006). El diálogo bibliotecología - sociología para el estudio de la relación bibliotecas y sociedad Paper presented at the Primer Coloquio de Bibliotecarios de la Biblioteca Vasconcelos, Ciudad de México.

Álvarez Álvarez, C. (2008). La etnografía como modelo de investigación en educación. Gazeta de antropología, 1(24).

Álvarez Pedrosian, E. (2010). Etnografías de la subjetividad. (Doctorado ), Facultad de Filosofía Barcelona

Ardèvol, E., Adolfo, E., \& Domínguez, D. (2008). La mediación tecnológica en la práctica etnográfica: Ankulegi antropologia elkartea.

Arquero Avilés, R. (2002). Estudios cualitativos sobre análisis y evaluación de la investigación en bilbioteconomía y documentación: Revisión bibliográfica Investigación bibliotecológica 16(32).

Bartolomé, M.A. (2003). En defensa de la etnografía. El papel contemporáneo de la investigación intercultural. Revista de Antropología Social, 12, 199-222.

Basail, A. (2002). Un acercamiento al objeto de estudio de la antropología Catauro. Revista cubana de antropología(5), 17-20.

Basail, A., \& Alvarez, D. (2004). Sociología de la cultura La Habana: Editorial Félix Varela.

Bicalho, L., \& Oliveira, M. (2011). Aspectos conceituais da transdisciplinaridade e a pesquisa em ciência da informação. Inf. \& Soc 21(2), 87-102.

Bourdieu, P. (1990). Sociología y cultura Mexico, D.F: Grijalbo.

Bufrem, L.S., \& Santos, S.d.F. (2009). Ciência da informação e uso metodológico da etnografia. Educação Temática Digital, 11(1), 148-174.

Calventus Salvador, J. (1996). Etnografia y psicologia social. Retrieved Enero, 2012, from http://sapiens.ya.com/estadistic/escrits/em.html

Campo, M.D.O. (2001). "Estar aqui" e "estar lá": Tensões e interseções com o trabalho de campo. Paper presented at the 10 Congresso Brasileiro de Etnomatemática.

Cheng, Q. (2008). Absence of audience's consciousness in ethnography on the writing of ethnography from an audience angle based on communication. Asian Social Science, 4(12).

Covarrubias Cuéllar, K.y. (1998). Etnografía: El registro del mundo social desde la vida cotidiana (apuntes metodológico) Estudios sobre las Culturas Contemporaneas, IV(008), 93-119.

Diaz de Rada, A. (2010). La lógica de la investigación etnográfica y la mediación computacional de la comunicación. Viejos problemas con un nuevo énfasis. Revista Chilena de Antropología Visual (15), 40-57

Díaz de Rada, Á. (1992). Etnociencia. El orden del sentido y el sentido del orden

Díaz de Rada, Á. (2003). Las formas del holismo.La construcción teórica de la totalidad en etnografía. Revista de Dialectología y Tradiciones Populares, 58(1). 
Domínguez Figaredo, D. (2007). Sobre la intención de la etnografía virtual. Revista Electrónica Teoría de la Educación. Educación y Cultura en la Sociedad de la Información. , 8(1).

Ferrada Cubillos, M. (2006). Etnografía un enfoque para la investigación de weblogs en biblioteconomía y documentación Biblios: Revista de Biblioteconomía y Documentación 7(023).

Firat, M. (2011). Virtual ethnography research on second life virtual communities. Turkish Online Journal of Distance EducationTOJDE 12

García Ferrando, M., Ibáñez, J., \& Alvira, F. (1994). El análisis de la realidad social. Métodos y técnicas de investigación. Madrid: Alianza.

García Mora, C. (2011). Escritura de la antropología. El delicioso suplicio de escribir antropología. Retrieved diciembre de 2011, from http://knol.google.com/k/carlos-garc\%C3\%ADa-mora/escritura-de-la-antropolog\%C3\%ADa/1dzkd4r4rdt65/8

Geertz, C. (1986). La interpretación de las culturas. Barcelona Gedisa SA.

Gil, L.M., Henao, C.M., \& Peñuela, A. (2004). Etnografía: Una visión desde la orientación analítica. 38.

Gómez, M.N.G.d. (2010). Metodologia de pesquisa no campo da ciência da informação DataGramaZero - Revista de Ciência da Informação, 1(6).

Herrero, J. (2002). ¿Qué es cultura? Retrieved Noviembre, 2011, from http://www.sil.org/capacitar/antro/cultura.pdf.

Hjørland, B. (2002a). Domain analysis in information science. Eleven approach-traditional as well as innovative. Journal of Documentation 58(4).

Hjørland , B. (2002b). La epistemología y la perspectiva socio cognitiva en la ciencia de la información. Journal of the American Society for Information Science and Technology, 53 (4).

Hjørland, B. (2004). Domain analysis: A socio-cognitive orientation for information science research. American Society for Information Science and Technology. Vol. 30, No. 3, 30(3).

Hjørland, B. (2007a). Domain analysis as approach to knowledge organization. Retrieved Diciembre de 2011, from http://www.iva.dk/bh/lifeboat ko/CONCEPTS/domain analysis.htm

Hjørland, B. (2007b). Domain analysis. Retrieved Enero 2012, from http://www.iva.dk/bh/lifeboat_ko/concepts/domain_analysis.htm

Hjørland, B., \& Albrechtsen, H. (1995). Hacia un nuevo horizonte en la ciencia de la información: El análisis de dominio. Journal of the American Society for Information Science and Technology, 46 (6).

Hjørland , B., \& Hartel , J. (2003). Afterword: Ontological, epistemological and sociological dimensions of domains. Knowledge Organization, 30(3/4), 239-245.

Hjørland, B., \& Nicolaisen, J. (2005). The epistemological lifeboat. Epistemology and philosophy of science for information scientists . Retrieved Enero de 2012, from http://www.db.dk/jni/lifeboat/home.htm

Hjørland, B., \& Nissen, K. (2005). Classification theory according to the domain-analytic point of view. Retrieved Noviembre 2011, from

http://www.db.dk/bh/lifeboat ko/CONCEPTS/Hj\%C3\%B8rland\%20\&\%20Nissen 2005 summary\%20of\%20arguments.htm

Jociles Rubio, M.I. (1999). Las técnicas de investigación en antropología. Mirada antropológica y proceso etnográfico. Gazeta de Antropología 15(1).

Kottak, C.P. (1997). Antropología cultural: Espejo para la humanidad. Madrid: McGraw-Hill

Marcus, G.E. (2001). Etnografía en/del sistema mundo. Alteridades 11, 111-127.

Martínez Miguélez, M. (2005). El método etnográfico de investigación Retrieved Diciembre 2011, from http://prof.usb.ve/miguelm/metodoetnografico.html

Martínez Miguélez, M. (2006). Investigación cualitativa. Sintesis conceptual IIPSI, 9(1), 123-146.

Matus Sepúlveda, G., \& Molina Luque, F. (2006). Metodología cualitativa. Un aporte de la sociología para investigar en bibliotecología. Valparaíso Universidad de Playa Ancha.

Mayernik, M. (2007). Evaluating domain analysis as a philosophical framework for Information Science. Retrieved Diciembre 2011, from http://polaris.gseis. ucla.edu/mmayerni/Birger Hj\%F8rland Paper.pdf

Meneses Tello, F. (2005). Bibliotecas y sociedad: Reflexiones desde una perspectiva sociológica. Revista Interamericana de Bibliotecología, 28(2), 123-. 
Moreno Fernández, M. (2007). Antropología y etnología. Análisis filosófico del término antropología y desarrollo del alcance gnoseológico de la etnología como ciencia. El Catoblepas(69).

Mosquera Villegas, M.A. (2008). De la etnografía antropológica a la etnografía virtual. Fermentum. Revista Venezolana de Sociología y Antropología, 18(53).

Muela Meza, Z.M. (2004). Una introducción a las metodologías de investigación cualitativa aplicadas a la bibliotecología. LIBER: Revista de Bibliotecología, 2(12).

Murillo, J., \& Martínez, C. (2010). Investigación etnográfica. Retrieved Noviembre 2011, from http://www.uam.es/personal pdi/stmaria/imurillo/InvestigacionEE/Presentaciones/Curso 10// Etnografica Trabajo.pdf

Nascimento, D.M., \& Marteleto, R.M. (2004). A “informação construída” nos meandros dos conceitos da teoria social de Pierre Bordieu DataGramaZero - Revista de Ciência da Informação 5(5).

Nivón, E., \& Rosas, A.M. (1991). Para interpretar a Clifford Geertz. Símbolos y metáforas en el análisis de la cultura Alteridades, 1(1), 40-49.

Prus, R. (2007). On studying ethnologs (not just people, societies in miniature) the necessities of ethnography, history, and comparative analysis. Journal of Contemporary Ethnography, 36(6).

Rappaport, J. (2007). Más allá de la escritura: La epistemología de la etnografía en colaboración. Revista Colombiana de Antropología, 43, 197-229

Rodríguez Gómez, G., Gil Flores, J., \& Garcia Jiménez, E. (1996). Metodologia de la investigacion cualitativa: Aljibe.

Rostagnol, S. (2011). Trabajo de campo en entornos diversos. Reflexiones sobre las estrategias de conocimiento. Gazeta de antropología, 1(27).

Sánchez Carretero, C. (2003). Voces y escritura: La reflexividad en el texto etnográfico. Revista de Dialectología y Tradiciones Populares, 58 (1).

Sanjek, R. (1990). The makings of anthropology. Ithaca, Estados Unidos de América: Cornell Editor.

Turpo Gebera, O.W. (2008). La netnografía Revista Iberoamericana de Educación (42).

Urbano Gil, H. (2007). El enfoque etnometodológico en la investigación científica. Liberabit, 13.

Urraco Solanilla, M. (2007). La metodología cualitativa para la investigación en ciencias sociales.Una aproximación "mediográfica". Revista sociológica de pensamineto crítico 1(1)

Vega Almeida, R.L., Fernández-Molina, J.C., \& Linares Columbié, R. (2009). Coordenadas paradigmáticas, históricas y epistemológicas de la ciencia de la información: Una sistematización. Information Research, 14(2).

Whitehead, T.L. (2005). Basic classical ethnographic research methods secondary data analysis, fieldwork, observation/participant observation, an informal and semi-structured interviewing. Retrieved Diciembre 2011, from http://www.cusag.umd.edu/documents/WorkingPapers/ClassicalEthnoMethods.pdf 


\section{Datos de los autores}

Manuel Alejandro Romero Quesada

Licenciado en Bibliotecología y Ciencia de la Información en 2012. Se encuentra realizando una Maestría en Bibliotecología y Ciencia de la Información. Profesor de la carrera Ciencias de la Información de la Facultad de Comunicación en la Universidad de La Habana.

manuel.romero@fcom.uh.cu

\section{Ania Rosa Hernández Quintana}

Doctor en Ciencias de la Información por la Universidad de La Habana, 2006. Profesora Principal Titular de la carrera Ciencias de la Información de la Facultad de Comunicación en la Universidad de La Habana.

aniahdez@fcom.uh.cu

Recibido - Received: 2015-09-25

Aceptado - Accepted: 2015-12-21

\section{$(\mathrm{cc}) \mathrm{BY}$}

This work is licensed under a Creative Commons Attribution 4.0

United States License.

\section{ULIS DDof}

This journal is published by the University Library System of the University of Pittsburgh as part of its D-Scribe Digital Publishing Program and is cosponsored by the University of Pittsburgh Press. 